\section{Necrotizing leptomeningeal vasculitis associated with a compressive meningioma in a cat: a rare paraneoplastic syndrome}

\author{
Les J. Gabor,1,2 Ines Walther, 1 \\ Kathleen Mullins, 3 Melinda Gabor, 4 \\ Andrea Bourque 1 \\ 1Department of Microbiology and \\ Pathology, Atlantic Veterinary College, \\ University of Prince Edward Island, \\ Charlottetown, PE, Canada; 2 Pre Clinical \\ Safety, Novartis Animal Health Australia, \\ Yarrandoo, Kemps Creek, NSW, Australia; \\ 3Veterinary Regional Referral Hospital, \\ Decatur, AL, USA; 4State Veterinary \\ Diagnostic Laboratory, EMAI, Menangle, \\ Australia
}

\section{Abstract}

A 17-year-old cat with a compressive meningioma was found to have an intradural, severe, necrotizing vasculitis, spatially un-associated with the neoplasm. Paraneoplastic vasculitis has been reported in two cases in the human literature associated with meningiomas. This is the first report of such an association in a domestic species.

\section{Introduction}

Vasculitis is broadly classified as primary or secondary, ${ }^{1}$ and, due their relative frequency and clinical significance, a specific classification system exists for categorizing primary vasculitides in humans. They remain, however, largely idiopathic in nature.2,3 Secondary vasculitides are generally associated with an infectious agent, or, non infectious causes such as autoimmune conditions or direct drug interactions. $^{3}$ One uncommon association with secondary vasculitis is malignancy. Paraneoplastic vasculitis, most usually associated with hematological malignancies such as leukemias, is most commonly cutaneous in manifestation. ${ }^{4}$ Cases of concurrent vasculitis associated with solid neoplasms are less well described - when reported, these are typically pulmonary, prostatic, colonic, renal or breast related carcinomas.5,6 Vasculitis associated with malignancy of the nervous tissue is extremely rare. In the case of meningiomas, two cases have been reported in the human literature: a single report associated with a subdural spinal meningioma, ${ }^{7}$ and a second case involving an intracranial meningioma. ${ }^{8}$ The veterinary literature tends to classify vasculitis in a fundamentally different manner, the principal categories being infectious versus non infectious. ${ }^{1}$ Of these, infectious causes are by far more numerous and include a number of significant diseases commonly encountered in veterinary species. In the cat, for example, Feline Corona Virus classically manifests as a vasculocentric inflammatory response. ${ }^{9}$ Non infectious causes of vasculitis in animals are relatively poorly defined, however, a number of well known conditions are described which have analogues in the human literature. These include beagle pain syndrome, which is well described. Beagle pain syndrome is often associated with anti-neutrophil cytoplasmic antibodies (ANCA) and is considered to be similar to the human condition, Kawasaki disease. ${ }^{10-13}$ The closely related steroid responsive meningitis / arteritis has many characteristics of an immune mediated disorder, however, it is considered idiopathic. 14,15

To date, there has been no publication in domestic or companion animals of vasculitis associated with malignancy.

\section{Case Report}

A 17-year-old domestic short haired cat presented to the Atlantic Veterinary College with a two day history of lethargy, weakness, anorexia and head pressing. The animal had a 4 year history of hyperthyroidism, which had been successfully controlled. During physical examination, anisocoria was noted, with left pupillary dilation. The animal continually paced, was non responsive to external stimuli, lacked a menace response on the left side, and failed to demonstrate a left sided direct or consensual papillary light reflex. Additionally, there were significant proprioceptive deficits in all four limbs and a lack of placing responses in the forelimbs. A preliminary diagnosis of cerebral hemorrhage, thromboembolism or central nervous neoplasia were all considered differential diagnoses, and the owners elected euthanasia.

At necropsy, the animal was in reasonable body condition, and minimal findings were found outside of the nervous system. There was a $2 \mathrm{~cm}$ diameter soft tissue protrusion on the ventral surface of the left piriform lobe, with compression of the cerebral cortex. At the level of the caudal brain stem and proximal cervical spinal cord, there was a focally expansile subdural hemorrhage. Histologically, the mass on the piriform lobe consisted of a focally expansile mass of spindle to irregular shaped cells, forming irregular whorls and bundles. The cells were moderately variable, with no mitoses noted. At the margins of the mass, there was a distinct zone of compression
Correspondence: Les J. Gabor, Pre Clinical Safety, Novartis Animal Health Australia, Yarrandoo, Kemps Creek, NSW, Australia

E-mail: les.gabor@novartis.com

Key words: paraneoplastic syndrome, vasculitis, feline, neuropathology.

Received for publication: 2 December 2011. Accepted for publication: 6 December 2011.

This work is licensed under a Creative Commons Attribution NonCommercial 3.0 License (CC BYNC 3.0).

(C) Copyright L.J. Gabor et al., 2012

Licensee PAGEPress srl, Italy

Veterinary Science Development 2012; 2:e4 doi:10.4081/vsd.2012.e4

of the cerebral cortex, and a focal line of hemorrhage. The findings were consistent with a meningioma. The brain stem and spinal cord junction contained an extensive zone of subdural hemorrhage, associated with severe fibrinoid necrotizing vasculitis of the dural blood vessels (Figure 1). The tunica intima of multiple vessels was replaced by a retractile, fibrinoid band. The remnant tunica media and adventitia, and surrounding connective tissue, were significantly infiltrated with abundant neutrophils, lymphocytes and plasma cells with lesser macrophages. Immunohistochemistry was performed for Feline Corona Virus, Toxoplasma gondii, Rabies and West Nile Virus antigens, with no positive findings.

\section{Discussion}

The presentation of this case, clinically, was strongly supportive of a focal, centrally located lesion, and was consistent with the demonstrated meningioma. The acute nature of presentation, however, was somewhat unusual. There was no evidence nor history of any traumatic incident. It is likely that the severe necrotizing vasculitis and focally extensive subdural hemorrhage had a significant effect on the presentation. The most commonly occurring infectious cases were excluded, and commercially available FIV and FeLV tests were found to be negative.

The vascular changes were histologically similar to those found in cases of idiopathic canine polyarteritis, which frequently presents with cranial cervical cord distribution. ${ }^{12}$ The advanced age of this cat, however, was in distinct contrast to the distribution of that condition, which is more common in far younger dogs. 14 The presence of severe vasculitis, spa- 


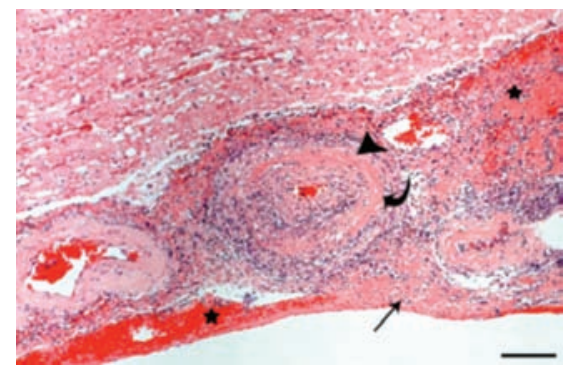

Figure 1. Feline subdural vasculitis. Within the markedly thickened dura a prominent, severely affected blood vessel (arrow head) is present. There is a dense band of refractile, fibrinoid material widening or effacing the normal intimal structure (curved arrow) containing abundant cellular debris. There are focal zones of hemorrhage (stars) and fibrin deposition (arrow). There is moderate malacia of the underlying white matter. HE. Scale bar: $100 \mu \mathrm{m}$.

tially at some distance to the meningioma, was considered to be consistent with a paraneoplastic origin.

Within human medicine, paraneoplastic vasculitides are uncommon, accounting for less than $5 \%$ all cases. ${ }^{16}$ The majority of cases are found concurrently with hematological malignancies; 6 however, solid malignancies are described. The presence of vasculitis associated with a meningioma has been reported in two single case reports in the human literature, 7,8 and as such, is exceedingly rare. In the two human cases, there was no spatial relationship between the tumours and the location of vasculitis. In the first case, an intracranial meningioma was associated with vasculitis of the aortic arch. In the second, a spinal benign meningioma located at $\mathrm{T} 1$ was associated with vasculitis of the coronary artery.

Paraneoplastic conditions are not unknown in the veterinary literature. Recently, myokymia was reported in association with an intracranial meningioma. ${ }^{17}$ Vasculitis, however, in conjunction with a solid neoplasm has to the knowledge of the authors, not been reported in any non human species.

\section{References}

1. Maxie G, Robinson W. The cardiovascular system. In: G. Maxie G (ed.) Pathology of domestic animals. Philadelphia, Elsevier Saunders, 2008. pp 1-107.

2. Hunder GG, Arend WP, Bloch DA, et al. The American-College-of-Rheumatology 1990 criteria for the classification of vasculitis. Introduction. Arthritis Rheum 1990;33: 1065-7.

3. Kallenberg CGM. The last classification of vasculitis. Clin Rev Allergy Immunol 2008;35:5-10.

4. Greer JM, Longley S, Edwards NL, et al. Vasculitis associated with malignancy. Experience with 13 patients and literature review. Medicine (Baltimore) 1988;67: 220-30.

5. Kurzrock R, Cohen PR, Markowitz A. Clinical manifestations of vasculitis in patients with solid tumors: a case report and review of the literature. Arch Intern Med 1994;154:334-40.

6. Solans-Laque R, Bosch-Gil JA, PerezBocanegra C, et al. Paraneoplastic vasculitis in patients with solid tumors: report of 15 cases. J Rheumatol 2008;35:294-304.
7. Al-Abbad AJA, Malleson PN, Petty RE, Cabral DA. Apparent medium vessel vasculitis associated with a spinal meningioma. J Rheumatol 1999;26:1211-2.

8. Canavero S, Pagni CA. Meningioma and Takayasu disease: case report. Ital J Neurol Sci 1990;11:393-4.

9. Kipar A, May H, Menger S, et al. Morphologic features and development of granulomatous vasculitis in feline infectious peritonitis. Vet Pathol 2005;42:321-30.

10. Albassam MA, Houston BJ, Greaves P, Barsoum N. Polyarteritis in a Beagle. J Am Vet Med Assoc 1989;194:1595-7.

11. Scottmoncrieff JC, Snyder PW, Glickman LT, Davis E, Felsburg PJ. Systemic necrotizing vasculitis in nine young beagles. $\mathrm{J}$ Am Vet Med Assoc 1992;201:1553-7.

12. Snyder PW, Kazacos EA, Scottmoncrieff JC, et al. Pathological features of naturally occurring juvenile polyarteritis in beagle dogs. Vet Pathol 1995;32:337-45.

13. Son W. Idiopathic canine polyarteritis in control beagle dogs from toxicity studies. J Vet Sci 2004;5:147-50.

14. Tipold A, Schatzberg SJ. An update on steroid responsive meningitis-arteritis. J Small Anim Pract 2010;51:150-4.

15. Wrzosek M, Konar M, Vandevelde M, Oevermann A. Cerebral extension of steroid-responsive meningitis arteritis in a boxer. J Small Anim Pract 2009;50:35-7.

16. Kurzrock R, Cohen PR. Vasculitis and cancer. Clin Dermat 1993;11:175-87.

17. Holland CT, Holland JT, Rozmanec M. Unilateral facial myokymia in a dog with an intracranial meningioma. Aust Vet J 2010;88:357-61. 